\title{
Synthesis of Cu@HBP-SH@Ag Particles by Using Thiol-Terminated Hyperbranched Polymer as Template and Dispersant
}

\author{
Wensong $\mathrm{Han}^{1} \cdot$ Qin Guo ${ }^{1}$ Tianhui Su${ }^{1}$
}

Received: 19 October 2020 / Accepted: 26 March 2021 / Published online: 16 April 2021

(c) The Minerals, Metals \& Materials Society 2021

\begin{abstract}
Cu@HBP-SH@Ag particles have been prepared by a facile synthesis strategy. Initially, thiol-terminated hyperbranched polymer (HBP-SH) was synthesized by stepwise polymerization. Subsequently, Cu particles covered with HBP-SH were prepared by reduction technology using HBP-SH as dispersant and template. Finally, Cu@HBP-SH@Ag particles were obtained by reduction reaction. Fourier-transform infrared (FT-IR) and ultraviolet-visible (UV-Vis) spectroscopy confirmed presence of HBP-SH on the surface of the $\mathrm{Cu}$ particles. The crystal structure was examined by $\mathrm{x}$-ray diffraction (XRD) analysis. Scanning electron microscopy (SEM) and energy-dispersive spectrometry (EDS) analysis confirmed formation of Cu@HBP-SH@Ag particles. Thermogravimetric analysis (TGA) showed that the Cu@HBP-SH@Ag particles had better antioxidation properties than the $\mathrm{Cu}$ particles.
\end{abstract}

Keywords Hyperbranched polymers·Cu@HBP-SH@Ag particles · template · synthesis

\section{Introduction}

Core-shell nanoparticles have attracted considerable attention due to their unique optical, electrical, and catalytic properties. They have been widely used in fields such as microelectronics, information materials, etc. ${ }^{1-4}$ In recent years, various kinds of core-shell nanoparticles have been prepared, among which Cu@Ag nanoparticles have received particular attention. As is well known, copper nanoparticles are easily oxidized at high temperature while silver exhibits good oxidation resistance. However, the lattice constants of $\mathrm{Ag}(0.361 \mathrm{~nm})$ and $\mathrm{Cu}(0.409 \mathrm{~nm})$ are different, and it is difficult to synthesize $\mathrm{Cu} @ \mathrm{Ag}$ nanoparticles using traditional methods. ${ }^{5,6}$

Hyperbranched polymers have attracted considerable attention in recent decades, offering a highly branched molecular structure and a large number of end groups and inner cavities, and thus exhibiting favorable solubility, low viscosity, less chain entanglement, and high chemical reactivity. These polymers have been widely used in various

Wensong Han

qluhws@163.com

1 School of Chemistry and Chemical Engineering, Qilu University of Technology (Shandong Academy of Sciences), Jinan 250353, People's Republic of China fields. ${ }^{7-9}$ Specifically, they have been used as templates to synthesize nanoparticles. Frangville et al. ${ }^{10}$ synthesized gadolinium phosphate nanowires under mild conditions based on poly(amidoamine). Sun et al. ${ }^{11}$ synthesized silver nanoparticles using a hyperbranched poly(amine ester) template. Mahapatra et al. ${ }^{12}$ synthesized silver nanoparticle in hyperbranched polyamine. In our previous studies, we synthesized Cu-Ag@Ag particles using hyperbranched polyester as template. ${ }^{13}$ To date, various approaches have been developed to synthesize core-shell nanoparticles, including wet chemical reduction and colloidal synthesis. ${ }^{14,15}$ However, few synthetic routes can yield Cu@ Ag by using thiolterminated hyperbranched polymers as a dispersant and modifying agent. This route is simple and effective since Ag binds strongly to thiol, which favors directional deposition of silver.

In the work described herein, a third-generation hyperbranched polyester (HBPE) was first synthesized then reacted with thioglycolic acid to synthesize HBP-SH. Cu particles were prepared by using HBP-SH as template and dispersant.Cu@HBP-SH@Ag particles were obtained by coating silver on the $\mathrm{Cu}$ particles by reduction of silver nitrate. Because of the chemical bonding between thiol and silver, the Cu@HBP-SH@Ag particles prepared by this method exhibit excellent antioxidant, electronic, and 
catalytic properties. They can be widely used in electronic pastes, as information storage and imaging materials, etc.

\section{Experimental Procedures}

\section{Materials}

Pentaerythritol (PE), mercaptoacetic acid, and methyl acrylate were purchased from Aladdin Reagent Co. (Shanghai, China). Copper nitrate $\left[\mathrm{Cu}\left(\mathrm{NO}_{3}\right)_{2} \cdot 5 \mathrm{H}_{2} \mathrm{O}, 99.5 \%\right]$, ascorbic acid, and silver nitrate were obtained from Sinopharm Chemical Reagent Co. Ltd, China. $N, N$-Dimethylformamide, ethanol, ammonia water, and $p$-toluenesulfonic acid ( $p$-TSA) were obtained from Ling Feng Chemical Reagent Co. Ltd. (Shanghai, China). All of these chemicals were used as received without further purification.

\section{Preparation of Third-Generation HBP-SH}

HBP-SH was prepared according to a three-step procedure. First, third-generation HBPE was synthesized as previously reported in literature. ${ }^{13}$ Then, HBPE $(3.707 \mathrm{~g}, 0.5 \mathrm{mmol})$, mercaptoacetic acid $(1.474 \mathrm{~g}, 16 \mathrm{mmol})$, and $50 \mathrm{ml} \mathrm{N}, \mathrm{N}-$ dimethylformamide were added to a $150-\mathrm{mL}$ three-necked flask equipped with a water separator, nitrogen inlet, thermometer, and magnetic stirrer. The mixture was heated at $80^{\circ} \mathrm{C}$ for $2 \mathrm{~h}$ in presence of $p$-TSA as catalyst. HBP-SH was obtained after dimethylacetamide was evaporated. Finally, the HBP-SH was refined by fractional precipitation. The synthesis process is presented in Fig. 1.

\section{Preparation of Cu Particles Using HBP-SH as Template and Dispersant}

HBP-SH $(1.00 \mathrm{~g})$ and $\mathrm{Cu}\left(\mathrm{NO}_{3}\right)_{2} \cdot 5 \mathrm{H}_{2} \mathrm{O}(0.25 \mathrm{~g} 1 \mathrm{mmol})$ were dissolved in $20 \mathrm{~mL}$ deionized water. Then, $12 \mathrm{~mL}$ aqueous solution of ascorbic acid $(0.1 \mathrm{M})$ was added to the mixture dropwise. The mixture was stirred for $1 \mathrm{~h}$. In this process, the $\mathrm{pH}$ of the solution was adjusted to between 4 and 5 using ammonia water. Finally, the $\mathrm{Cu}$ particles were filtrated and dried at $50^{\circ} \mathrm{C}$ for $6 \mathrm{~h}$ in vacuum, yielding $\mathrm{Cu}$ particles covered with HBP-SH, denoted as Cu@HBP-SH.

\section{Synthesis of Cu@HBP-SH@Ag Particles Using Cu Particles Covered with HBP-SH}

Cu@HBP-SH@Ag particles were synthesized by using $\mathrm{Cu}$ particles covered with HBP-SH. The typical process for synthesizing Cu@HBP-SH@Ag particles is as follows: $2.00 \mathrm{~g} \mathrm{Cu}$ particles and $\mathrm{Ag}\left(\mathrm{NO}_{3}\right)_{2}$ solution $(10 \mathrm{~mL}, 0.1 \mathrm{M})$ were dispersed in $20 \mathrm{~mL}$ distilled water, then the mixture was stirred for $30 \mathrm{~min}$. While stirring, $12 \mathrm{~mL}$ ascorbic acid
$(0.1 \mathrm{M})$ was added to the mixture dropwise. In this process, the $\mathrm{pH}$ value was kept between 4 and 5 by using ammonia water. The mixture was stirred for another 2 h. The $\mathrm{Cu} @$ HBP-SH@Ag particles were filtrated, then washed with water and ethanol for three times. Finally, the Cu@HBPSH@ Ag particles were dried at $50^{\circ} \mathrm{C}$ for $6 \mathrm{~h}$ in a vacuum. The synthesis process is presented in Fig. 2.

\section{Characterization}

Fourier-transform infrared (FT-IR) spectroscopy was carried out using a Nicolet Magna IR650 (Madison, WI). ${ }^{1} \mathrm{H}$ nuclear magnetic resonance (NMR) spectroscopy was carried out using a $300-\mathrm{MHz}$ instrument (Bruker). Ultraviolet-visible (UV-Vis) measurements were conducted using a Shimadzu 2450 spectrophotometer. X-Ray diffraction (XRD) patterns were obtained using a Bruker $\mathrm{D}_{8}$-ADVANCE powder $\mathrm{x}$-ray diffractometer. Scanning electron microscopy (SEM) images were obtained using a Sirion field-emission SEM. Thermogravimetric analysis (TGA) was performed on a TGA 1500 DSP-SF instrument at a heating rate of $10^{\circ} \mathrm{C} / \mathrm{min}$ under air atmosphere.

\section{Results and Discussion}

\section{FT-IR and ${ }^{1} \mathrm{H}$ NMR results}

Figure 3 shows the FT-IR spectra of (a) HBPE, (b) HBP$\mathrm{SH}$, (c) Cu@HBP-SH, and (d) Cu@HBP-SH@Ag. The characteristic peaks of $\mathrm{Cu}$ particles coated by HBP-SH are marked. In Fig. 3c, the peak at $3420 \mathrm{~cm}^{-1}$ is assigned to $\mathrm{O}-\mathrm{H}$ stretching vibration of HBP-SH, indicating presence of unesterified hydroxyl groups in HBP-SH. The band observed at around $2942 \mathrm{~cm}^{-1}$ is assigned to stretching vibration of $-\mathrm{CH}_{2}$ and $-\mathrm{CH}_{3}$. The peak seen at $2576 \mathrm{~cm}^{-1}$ is characteristic of $-\mathrm{SH}$. The peak observed at around $2682 \mathrm{~cm}^{-1}$ corresponds to stretching vibration of $\mathrm{S}-\mathrm{CH}_{2}$. The strong peak at $1720 \mathrm{~cm}^{-1}$ is assigned to carbonyl bond of ester groups. The peaks observed at $1154 \mathrm{~cm}^{-1}$ and $1048 \mathrm{~cm}^{-1}$ are assigned to symmetric stretching of $\mathrm{C}-\mathrm{O}$ and asymmetric stretching of HBP-SH. The reaction of mercaptoacetic acid with HBPE was further confirmed by ${ }^{1} \mathrm{H}-\mathrm{NMR}$. Figure 4 shows the ${ }^{1} \mathrm{H}-\mathrm{NMR}$ spectrum of HBP-SH. The chemical shift observed at $1.5 \mathrm{ppm}$ (Fig. 4, peak a) indicates the presence of $-\mathrm{SH}$ group. Proton signals were also observed at $3.5 \mathrm{ppm}$ to $4.0 \mathrm{ppm}\left(-\mathrm{OOC}-\mathrm{CH}_{2}-\mathrm{S}-\right.$ and $-\mathrm{CH}_{2}-\mathrm{N}<$, Fig. 4, peaks $\mathrm{b}$ and e), $4.3 \mathrm{ppm}\left(-\mathrm{C}-\mathrm{CH}_{2}-\mathrm{O}-\right.$, Fig. 4 , peak c), $2.9 \mathrm{ppm}$ $\left(-\mathrm{N}-\mathrm{CH}_{2}-\right.$, Fig. 4, peak d), and $2.5 \mathrm{ppm}\left(-\mathrm{OOC}-\mathrm{CH}_{2}\right.$, Fig. 4, peak f). These results confirm the coating of HBPSH on the surface of the copper particles. ${ }^{16,17}$ 

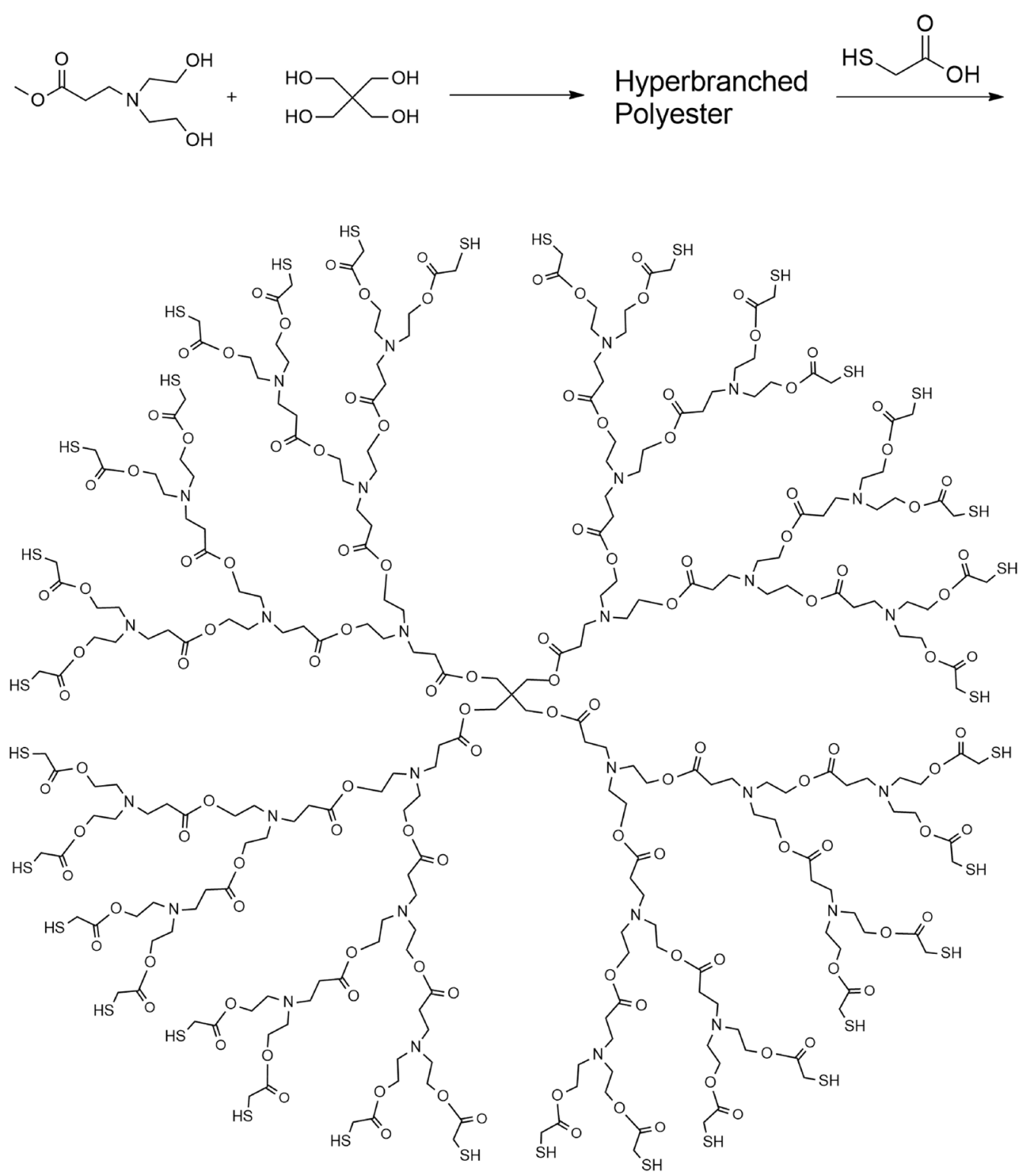

Fig. 1 Synthesis of third-generation HBP-SH.

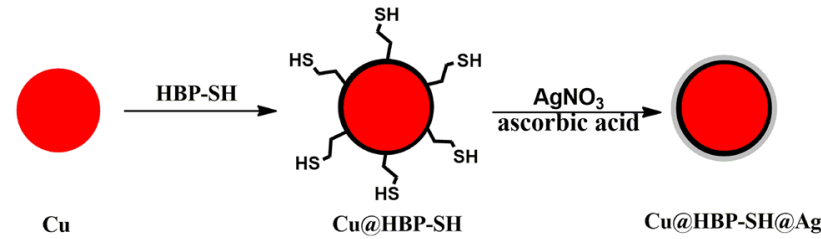

Fig. 2 Synthesis of Cu@HBP-SH@Ag particles using $\mathrm{Cu}$ particles covered with HBP-SH.

\section{UV-Vis Spectra}

Figure 5 shows the UV-Vis spectra of HBP-SH (a), Cu@ HBP-SH (b), HBPE (c), and Cu@HBP-SH@Ag (d).
It can be seen that the spectral profiles of HBP-SH (a) and HBPE (c) are similar. They all show sharp absorption bands at around $210 \mathrm{~nm}$. The molecular structures of HBPE and HBP-SH contain ester groups with a conjugated carbon-oxygen double bond $(\mathrm{C}=\mathrm{O})$. These can produce $\pi^{*}-\pi^{*}$ electronic transitions. ${ }^{18,19} \mathrm{Cu} @$ HBP-SH (Fig. 5b) also shows a sharp absorption at around $205 \mathrm{~nm}$, confirming that the surface of the $\mathrm{Cu}$ particles was coated with HBP-SH. After reaction with Ag, a new absorption peak appeared at around $425 \mathrm{~nm}$ for the Cu@HBP-SH@Ag (Fig. 5d) particles. This peak corresponds to the surface plasmon resonance absorption of the silver metal particles. As is known, Ag nanoparticles show an intense plasmon 


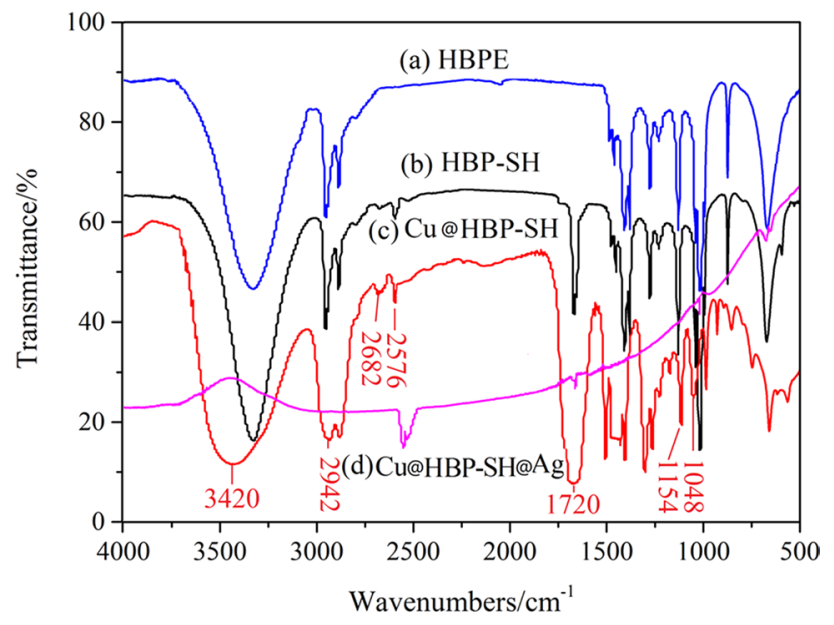

Fig. 3 FT-IR spectra of (a) HBPE, (b) HBP-SH, (c) Cu@HBP-SH, and (d) Cu@HBP-SH@Ag.

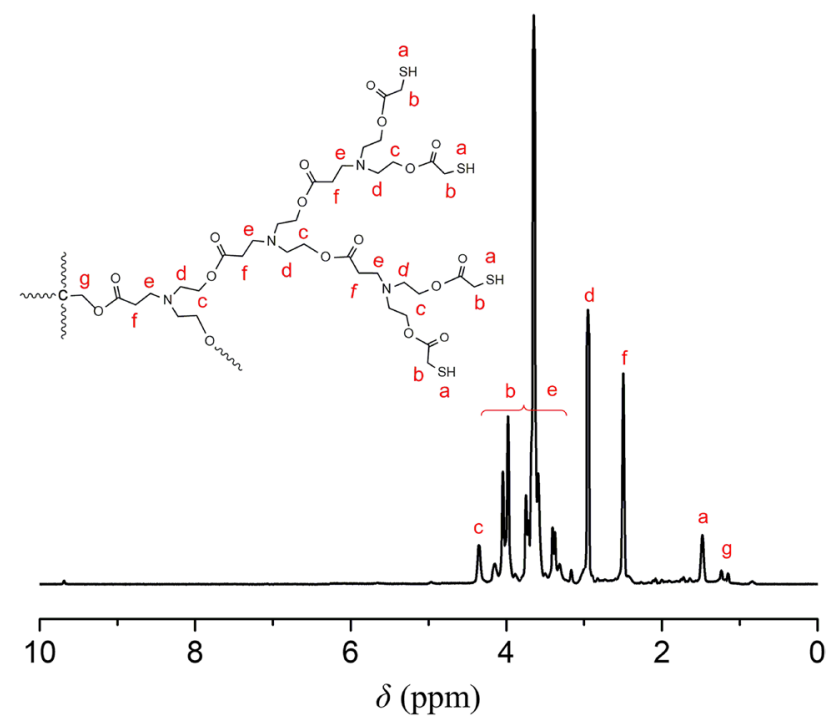

Fig. $4{ }^{1} \mathrm{H}-\mathrm{NMR}$ spectrum of HBP-SH.

absorption band in this region. This analysis confirms that the Ag particles were coated on the copper.

\section{XRD Analysis}

Figure 6 shows the XRD patterns of Cu@HBP-SH (a) and Cu@HBP-SH@Ag (b) particles, clearly showing peaks characteristic of $\mathrm{Cu}$ particles: $\mathrm{Cu}\left(\begin{array}{lll}1 & 1 & 1\end{array}\right)$ orientation at $43.2^{\circ}$,

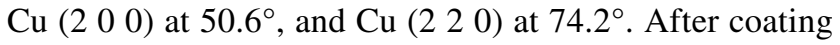
the $\mathrm{Cu}$ particles with $\mathrm{Ag}$, the characteristic peaks of $\mathrm{Ag}$ were detected: $\mathrm{Ag}\left(\begin{array}{lll}1 & 1 & 1\end{array}\right)$ orientation at $38.2^{\circ}, \mathrm{Ag}(200)$ at $44.4^{\circ}$, $\mathrm{Ag}(220)$ at $64.6^{\circ}$, and $\mathrm{Ag}(311)$ at $77.6^{\circ}$ (Fig. 6b). These peaks are consistent with literature values. ${ }^{20,21}$ Because Ag was completely coated on the surface of $\mathrm{Cu}$, the diffraction

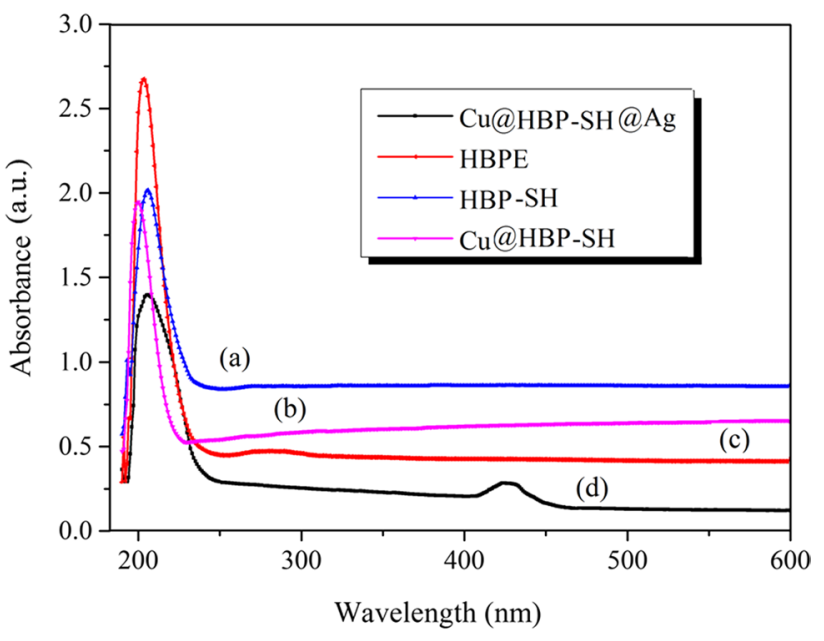

Fig. 5 UV-Vis spectra of HBP-SH (a), Cu@HBP-SH (b), HBPE (c), and Cu@HBP-SH@Ag (d) in ethanol.

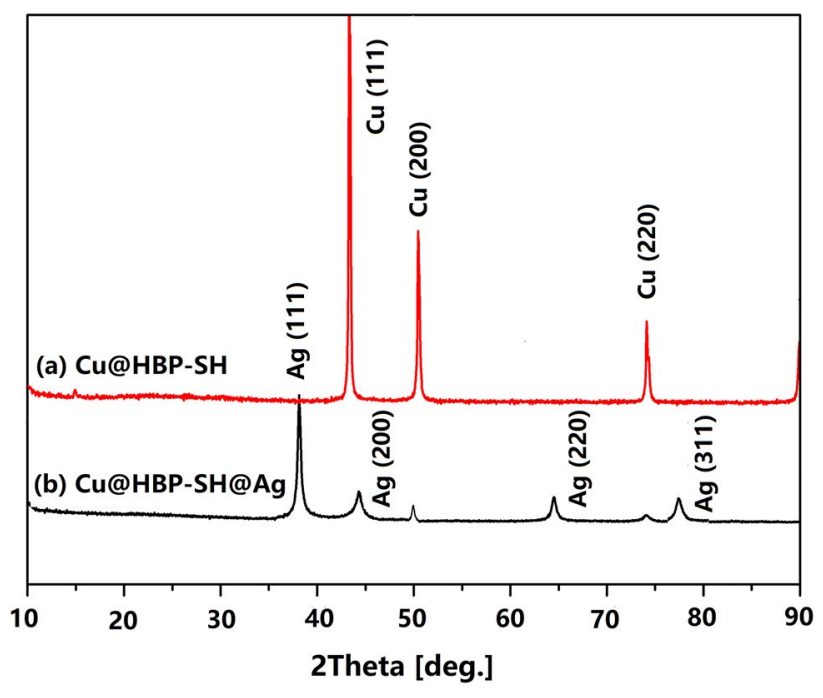

Fig. 6 XRD patterns of Cu@HBP-SH and Cu@HBP-SH@Ag.

peaks were dominated by the Ag particles. These XRD results show that the $\mathrm{Cu} @$ HBP-SH@Ag particles were successfully synthesized.

\section{SEM-EDX}

Figure 7 shows the SEM-EDX images of the Cu@HBP-SH and Cu@HBP-SH@Ag particles. Figure 7a shows that the Cu@HBP-SH particles had a spherical structure with diameter of $1 \mu \mathrm{m}$ to $2 \mu \mathrm{m}$. The elemental composition of the $\mathrm{Cu} @$ HBP-SH particles were measured by EDX (Fig. 7b), revealing signals for $\mathrm{Cu}, \mathrm{C}, \mathrm{O}$, and $\mathrm{S}$. These results indicate that the surface of copper was covered with HBP-SH. Figure 7c shows a SEM image of Cu@HBP-SH@Ag, revealing that 

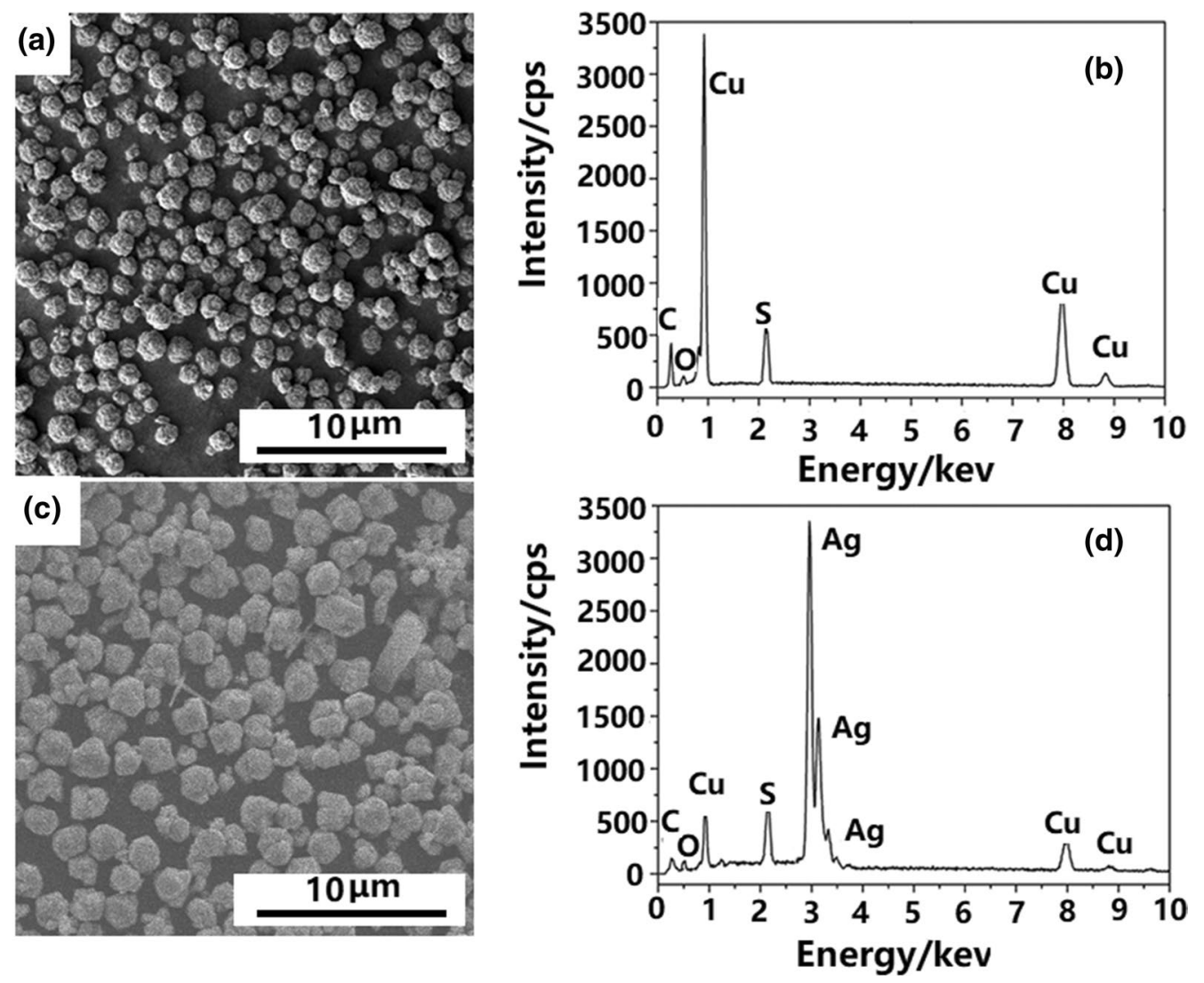

Fig.7 SEM images for Cu@HBP-SH (a) and Cu@HBP-SH@Ag (c); EDX spectra for Cu@HBP-SH (b) and Cu@HBP-SH@ Ag (d).

Cu@HBP-SH@Ag particles with a smooth surface were obtained after Ag aggregation. Compared with Cu@HBPSH, the particle size of Cu@HBP-SH@Ag was larger. All these results confirm the deposition of $\mathrm{Ag}$ on the surface of the Cu@HBP-SH particles. Figure 7d shows the EDX spectrum of Cu@HBP-SH@Ag. The EDX spectra confirmed the presence of elements $\mathrm{Ag}, \mathrm{Cu}, \mathrm{C}, \mathrm{O}$, and $\mathrm{S}$, further indicating the formation of Cu@HBP-SH@Ag particles.22,23

\section{TGA}

The thermal properties of the samples were characterized by TGA in air atmosphere. Figure 8 shows the TGA curves of HBPE, HBP-SH, Cu@HBP-SH, and Cu@HBP-SH@Ag. It can be seen that the HBPE and HBP-SH showed similar TGA curves and all the samples showed two-step degradation profiles (Fig. 8A, a and b). The first weight loss of around $60 \%$ occurred between around $250^{\circ} \mathrm{C}$ and $350^{\circ} \mathrm{C}$, being due to decomposition of outer terminal groups. The second weight loss of about $30 \%$ occurred in the temperature range from $350^{\circ} \mathrm{C}$ to $500^{\circ} \mathrm{C}$. This can be attributed to decomposition of the core of the hyperbranched polymer. It can also be seen that the decomposition onset temperature of HBPE was higher than that of HBP-SH. As is known, the decomposition temperature of hyperbranched polymers depends on their terminal groups and molar mass. Although the molecular weight of HBP-SH is larger than that of HBP, the HBPE has a large number of $\mathrm{OH}$ groups, which favor the formation of strong hydrogen bonds between molecules. Therefore, HBPE shows better thermal stability than HBPESH. ${ }^{24,25}$ Figure 8B shows the TGA curves of $\mathrm{Cu} @ \mathrm{HBP}-\mathrm{SH}$ and Cu@HBP-SH@Ag particles, which show increasing parts for Cu@HBP-SH and Cu@HBP-SH@Ag because of the oxidation of copper. For Cu@HBP-SH and Cu@HBPSH@Ag, weight loss occurs below $300^{\circ} \mathrm{C}$, which can be attributed to decomposition of HBP-SH. Weight increases of 9\% and 2\% can also be observed for Cu@ HBP-SH and Cu@HBP-SH@Ag, respectively (Fig.8 B, c and d). This indicates that the Cu@HBP-SH@Ag particles showed better antioxidation properties than $\mathrm{Cu}^{26}$

\section{Conclusions}

$\mathrm{Cu}$ particles covered with HBP-SH were prepared by using HBP-SH as template and dispersant. Using the chemical bond between SH and Ag, Cu@HBP-SH@Ag particles were then prepared. The FT-IR and UV-Vis spectra of the 

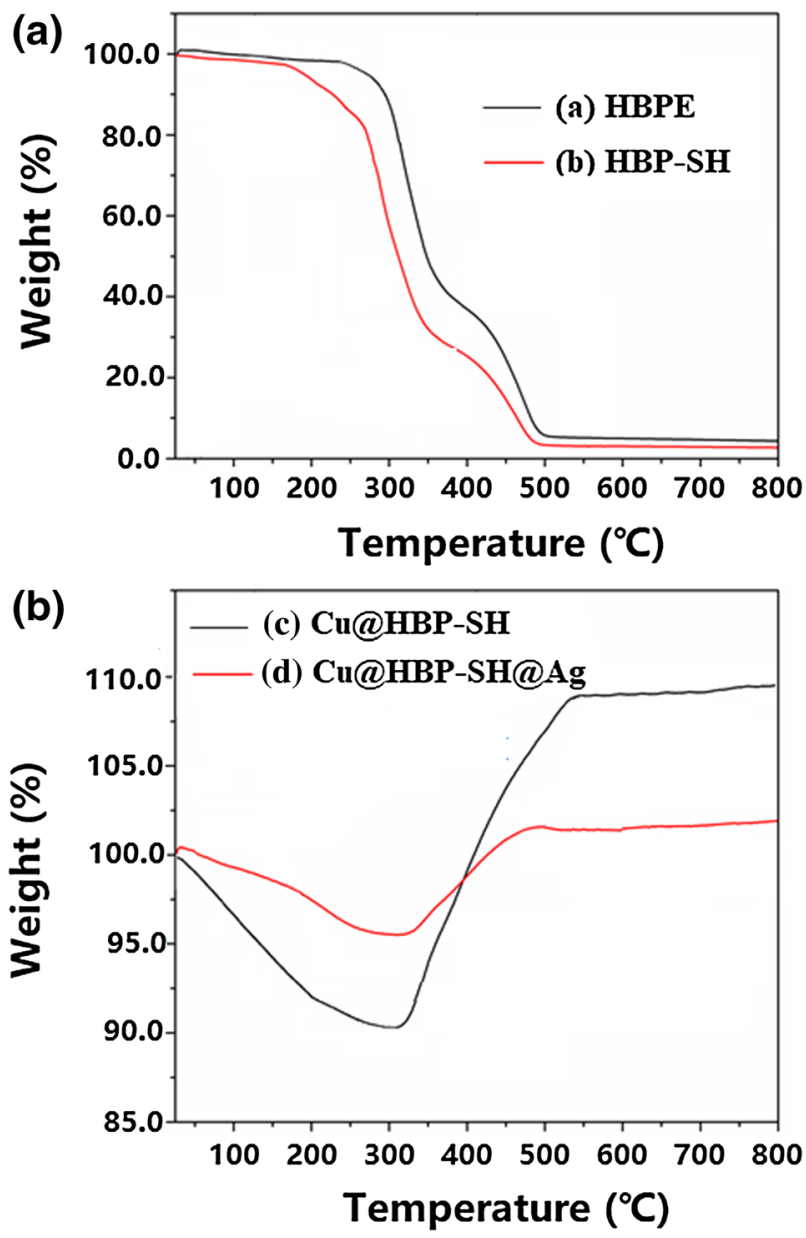

Fig. 8 TGA curves of (a) HBPE, (b) HBP-SH, (c) Cu@HBP-SH, and (d) Cu@HBP-SH@Ag.

samples confirmed that the surface of the $\mathrm{Cu}$ particles was coated with HBP-SH. XRD and SEM results confirmed the formation of $\mathrm{Cu} @ \mathrm{HBP}-\mathrm{SH} @ \mathrm{Ag}$ particles. Moreover, the Cu@HBP-SH@Ag particles showed better antioxidation properties than $\mathrm{Cu}$. The synthesized Cu@HBP-SH@ Ag particles have potential applications in electronic pastes, optoelectronic devices, etc.

Funding This study was funded by National College Students Innovation and Entrepreneurship Training Program (201710431023).

Conflict of interest The authors declare that they have no conflicts of interest.

\section{References}

1. L.L. Song, K. Mao, X.D. Zhou, and J.M. Hu, Talanta 146, 285 (2016).
2. M.J. Chen, Y.R. He, X.Z. Wang, and Y.W. Hu, Appl. Energ. 211, 735 (2018).

3. S.M. Majhi, G.K. Naik, H.J. Lee, H.G. Song, C.R. Lee, I.H. Lee, and Y.T. Yu, Sensors. Actuators. B Chem. 268, 223 (2018).

4. F. Fabris, E. Lima, E. De Biasi, H.E. Troiano, M.V. Mansilla, T.E. Torres, R.F. Pacheco, M.R. Lbrarra, G.F. Goya, R.D. Zysler, and E.L. Winkler, Nanoscale 11, 3164 (2019).

5. J.P. Wang, S.H. Shin, and A.M. Hu, J. Phys. Chem. C 120, 17791 (2016).

6. X. Yu, J.T. Li, T.L. Shi, C.L. Cheng, G.L. Liao, J.H. Fan, T.X. Li, and Z. Tang, J. Alloys Compd. 724, 365 (2017).

7. H. Chen and J. Kong, Polym. Chem. 7, 3643 (2016).

8. S.F. Chen, Z.J. Xu, and D.H. Zhang, Chem. Eng. J. 343, 283 (2018).

9. A.B. Cook, R. Barbey, J.A. Burns, and S. Perrier, Macromolecules 49, 1296 (2016).

10. C. Frangville, M. Gallois, Y.C. Li, H.H. Nguyen, N.L. Viguerie, D.R. Talhum, C. Mingotaud, and J.D. Marty, Nanoscale 8, 4252 (2016).

11. R. Sun, H. Zhao, Y. Luo, and Y. Liu, Nanopart. Res. 13, 1133 (2011).

12. S.S. Mahapatra and N. Karak, Mater. Chem. Phys. 112, 1114 (2008).

13. W.S. Han, J. Electron. Mater. 46, 7 (2017).

14. M.B. Gawande, A. Goswami, F.X. Felpin, T. Asefa, X.X. Huang, R. Silva, X.X. Zou, R. Zboril, and R.S. Varma, Chem. Rev. 116, 3722 (2016).

15. L.Y. Wei, J.G. Lu, H.Z. Xu, A. Patel, Z.S. Chen, and G.F. Chen, Drug. Discov. Today. 20, 595 (2015).

16. L.P. Wang, Y.Y. Liang, Q. Yu, S.S. Chen, J.H. Zhang, M.H. Miao, and D.H. Zhang, Polym. Compos. 39, 2046 (2018).

17. D.H. Zhang, C.H. Liu, S.F. Chen, J.H. Zhang, J. Cheng, and D.H. Zhang, Prog. Org. Coat. 101, 178 (2016).

18. W. Han, B. Lin, H. Yang, and X. Zhang, J. Appl. Polym. Sci. 128, 4261 (2013).

19. W.S. Han, B.P. Lin, H. Yang, and X.Q. Zhang, Polym. Bull. 68, 1009 (2012).

20. C.H. Tsai, S.Y. Chen, J.M. Song, I.G. Chen, and H.Y. Lee, Corros. Sci. 74, 123 (2013).

21. Y.H. Peng, C.H. Yang, K.T. Chen, S.R. Popuri, C.H. Lee, and B.S. Tang, Appl. Surf. Sci. 263, 38 (2012).

22. H. Li, C.Y. Guo, and C.L. Xu, Biosens. Bioelectron. 63, 339 (2015).

23. W.S. Han, J. Electron. Mater. 46, 4676 (2017).

24. J.M. Giussi, O. Azzaroni, S.H. Bielowka, Z. Wojnarowska, J. Knapik, and M. Paluch, Polymer 100, 227 (2016).

25. A. Matta, I. Katada, J.J. Kawazoe, P. Chammingkwan, M. Terano, and T. Taniike, Polym. Degrad. Stabi. 142, 50 (2017).

26. S.Q. Liu, B. Zhao, L. Jiang, Y.W. Zhu, X.Z. Fu, R. Sun, J.B. Xu, and C.P. Wang, J. Mater. Chem. C 6, 257 (2018).

Publisher's Note Springer Nature remains neutral with regard to jurisdictional claims in published maps and institutional affiliations. 